05

\title{
Влияние кобальта на кристаллическую структуру и магнетизм электрон-допированного оксида $\mathrm{Sr}_{0.8} \mathrm{Ce}_{0.2} \mathrm{MnO}_{3}$
}

\author{
(C) Г.В. Базуев ${ }^{1}$, Т.И. Чупахина ${ }^{1}$, А.В. Королев ${ }^{2}$ \\ ${ }^{1}$ Институт химии твердого тела УрО РАН, \\ Екатеринбург, Россия \\ ${ }^{2}$ Институт физики металлов им. М.Н. Михеева УрО РАН, \\ Екатеринбург, Россия \\ E-mail: bazuev@ihim.uran.ru
}

(Поступила в Редакцию 14 июня 2018 г.)

\begin{abstract}
Сложные оксиды $\mathrm{Sr}_{0.8} \mathrm{Ce}_{0.2} \mathrm{Mn}_{1-y} \mathrm{Co}_{y} \mathrm{O}_{3-\delta}(y=0.2,0.3,0.4,0.5,0.6)$ со структурой перовскита, полученные из простых оксидов методом твердофазных реакций, исследованы методами структурного анализа и измерений магнитных свойств. Замещение $\mathrm{Mn}$ на Со в разбавленном антиферромагнетике $\mathrm{Sr}_{0.8} \mathrm{Ce}_{0.2} \mathrm{MnO}_{3}$ (температура Нееля $T_{N}=210 \mathrm{~K}$ ) приводит к уменьшению степени тетрагонального искажения кристаллической структуры и к переходу к кубической ячейке. Наблюдаемое на первом этапе замещения Мn на Со вырождение антиферромагнитного взаимодействия $\left(T_{N}=138 \mathrm{~K}\right.$ при $\left.y=0.2\right)$ сменяется постепенным его усилением с возрастанием температуры магнитного превращения до $239 \mathrm{~K}$ при $y=0.6$. Увеличение содержания Со снижает конкуренцию между ферромагнитным и антиферромагнитным взаимодействиями и уменьшает температуру перехода в спин-стекольное состояние. Магнитная негомогенность и образование ферромагнитных кластеров $\mathrm{Co}^{2+}-\mathrm{Mn}^{4+}$ наблюдались в $\mathrm{Sr}_{0.8} \mathrm{Ce}_{0.2} \mathrm{Mn}_{0.4} \mathrm{Co}_{0.6} \mathrm{O}_{2.69}$ при $140 \mathrm{~K}$.
\end{abstract}

DOI: 10.21883/FTT.2018.12.46729.195

\section{1. Введение}

Манганиты и кобальтиты щелочноземельных и редкоземельных элементов с перовскитоподобной структурой постоянно привлекают внимание исследователей в связи с присущими им интересными и практически важными физическими и химическими свойствами. В частности, интерес к манганитам $L n_{1-x} A_{x} \mathrm{MnO}_{3}$ (где $L n-$ редкоземельный и $A$ - щелочноземельный элементы) вызван эффектом колоссального магнитного сопротивления, обусловленного оптимальным балансом междуспиновым, зарядовым и орбитальным упорядочением [1,2]. Кобальтит $\mathrm{SrCoO}_{3}-$ ферромагнетик (температура Кюри $T_{C}=270-300 \mathrm{~K}$ ) с металлической проводимостью [3], в то время как замещенный кобальтит $L a_{1-x} \mathrm{Sr}_{x} \mathrm{CoO}_{3}$ имеет сложную фазовую диаграмму с антиферромагнетизмом и изоляторным поведением при низких концентрациях $\mathrm{Sr}$, и ферромагнетизмом и проводящими свойствами при высоких.

В работах [4-6] исследовано влияние замещения $\mathrm{Mn}$ на Со в сложных оксидах $\mathrm{La}_{1-x} A_{x} \mathrm{Mn}_{1-y} \mathrm{Co}_{y} \mathrm{O}_{3}(A=\mathrm{Ca}$, $\mathrm{Sr})$ с дырочной проводимостью. Установлено, что рост содержания Со приводит к ослаблению ферромагнитного упорядочения и образованию спин стекольного состояния кластерного типа внутри ферромагнитного состояния. В частности, магнетизм перовскита $\mathrm{La}_{1.25} \mathrm{Sr}_{0.75} \mathrm{MnCoO}_{6}$ был классифицирован на основе двух превращений: ферромагнитного перехода при $T_{C}(217 \mathrm{~K})$ и зависимого от частоты перехода при $\mathrm{Tg}<100 \mathrm{~K}$ [6]. Высокая $T_{C}$ определяется локальными $T_{C}$ слабо флуктуирующих кластеров, сформированных на основе валентной комбинации $\mathrm{Co}^{2+}-\mathrm{Mn}^{4+}$, в то время как низкий спонтанный момент при $2 \mathrm{~K}$ является следствием их малого содержания.

Представляет интерес исследование влияния кобальта на структуру и свойства электрон допированных манганитов, в частности, твердых растворов (ТР) $A_{1-x} \mathrm{Ce}^{4+x} \mathrm{MnO}_{3}(A-\mathrm{Sr}, \mathrm{Ca})$ [7-9], которые рассматриваются как потенциальные катодные материалы для твердотельных топливных элементов [10]. Се-замещенные кобальтиты $A_{1-x} \mathrm{Ce}_{x}^{4+} \mathrm{CoO}_{3}$ интересны с точки зрения влияния гетеровалентного замещения на температуру Кюри и проводимость $[11,12]$.

Замещение в $\mathrm{SrMnO}_{3}$ и $\mathrm{SrCoO}_{3}$ стронция на высоковалентные катионы церия $\mathrm{Ce}^{4+}$ оказывает существенное влияние на их структурные и зарядовые характеристики, магнитные и транспортные свойства. Известно [13], что $\mathrm{SrMnO}_{3}$, полученный при обычных условиях, имеет гексагональную структуру и является антиферромагнетиком с температурой Нееля $T_{N}=278 \mathrm{~K}$. Введение высоковалентных катионов $\mathrm{Ce}^{4+}$ в позиции $\mathrm{Sr}$ сначала (при $x=0.01-0.05)$ стабилизирует кубическую структуру $\mathrm{Sr}_{1-x} \mathrm{Ce}_{x} \mathrm{MnO}_{3}$, а при $0.1<x \leq 0.3$ - структуру тетрагонально искаженного перовскита (пространственная группа $I 4 / \mathrm{mcm}$ ). При этом происходит восстановление соответствующего количества катионов $\mathrm{Mn}^{4+}$ до $\mathrm{Mn}^{3+}$, что приводит к сильному искажению октаэдров $\mathrm{MnO}_{6}$ и увеличению параметра $a$ и объема элементарной ячейки. Параметр $c$ в $\mathrm{Sr}_{1-x} \mathrm{Ce}_{x} \mathrm{MnO}_{3}$ сначала возрастает, достигая максимального значения при $x=0.15$ и в дальнейшем уменьшается [14].

Магнитные и электрические свойства данных ТР в значительной степени зависят от $x$. Твердый раствор 
$\mathrm{Sr}_{0.9} \mathrm{Ce}_{0.1} \mathrm{MnO}_{3}$ является антиферромагнетиком С-типа с $T_{N}=290 \mathrm{~K}$ и переходит из металлического состояния в полупроводниковое при $315 \mathrm{~K}$. Магнитное поведение $\mathrm{Sr}_{1-x} \mathrm{Ce}_{x} \mathrm{MnO}_{3}$ при $0.1<x \leq 0.3$ обусловлено сильной конкуренцией между двойным обменом и сверхобменным взаимодействием [9], результатом которой является отсутствие в данной системе магнитоупорядоченного состояния и доминирование при низких температурах (ниже $20 \mathrm{~K}$ ) кластер или спин-стекольного состояния. Температурная зависимость магнитной восприимчивости образца $\mathrm{Sr}_{0.8} \mathrm{Ce}_{0.2} \mathrm{MnO}_{3}$ демонстрирует широкий максимум при 150-300 K, который интерпретируется в рамках концепции разбавленного антиферромагнетизма с $T_{N}=210 \mathrm{~K}$. В парамагнитной области при $x \geq 0.2$, согласно [8], происходит образование ферромагнитных кластеров.

В твердых растворах со значением $x$, равным 0.25 и 0.35 [9], проявляется отрицательный магнеторезистивный эффект, в основе которого лежит зарядовое упорядочение, возникающее около $110 \mathrm{~K}$.

В работе [15] приводятся сведения о синтезе, строении и проводящих свойствах сложного оксида $\mathrm{Sr}_{0.8} \mathrm{Ce}_{0.2} \mathrm{Mn}_{0.8} \mathrm{Co}_{0.2} \mathrm{O}_{3}$. В отличие от $\mathrm{Sr}_{0.8} \mathrm{Ce}_{0.2} \mathrm{MnO}_{3}$, октаэдры в структуре Со-содержащего соединения являются регулярными, что связано с отсутствием ЯнТеллеровских катионов $\mathrm{Mn}^{3+}$. Введение кобальта на позиции Mn уменьшает электропроводность. Электрохимические характеристики $\mathrm{Sr}_{0.8} \mathrm{Ce}_{0.2} \mathrm{MnO}_{3}$, допированного кобальтом, как катодного материала приведены в работе [10].

Кристаллическая структура $\mathrm{Sr}_{1-x} \mathrm{Ce}_{x} \mathrm{CoO}_{3-\delta}$, полученного в условиях высокого давления [11], остается кубической вплоть до $x=0.4$. В случае синтеза в запаянных ампулах диапазон концентраций кубической фазы ограничен $0<x<0.15$ [12]. Этот ТР имеет кристаллическую структуру высокотемпературной модификации $\mathrm{SrCoO}_{3-x}$ и характеризуется высоким кислородным обменом и наличием электрической проводимости. Дефицит по кислороду соединения $\mathrm{Sr}_{1-y} \mathrm{Ce}_{y} \mathrm{CoO}_{3-\delta}$ увеличивается с температурой и уменьшается с ростом парциального давления кислорода $\mathrm{O}_{2}$ и с увеличением концентрации Се.

В настоящей работе представлены результаты исследования структурных характеристик и магнитных свойств ТР двойного замещения $\mathrm{Sr}_{0.8} \mathrm{Ce}_{0.2} \mathrm{Mn}_{1-y} \mathrm{Co}_{y} \mathrm{O}_{3-\delta} \quad$ в широкой области концентраций ( $y$ принимает значения $0.2,0.3,0.4,0.5$ и 0.6 ). В предыдущей работе [16], посвященной исследованию двух образцов из этой серии (с $y$, равным 0.3 и 0.4 ) было показано, что введение кобальта на позиции марганца в $\mathrm{Sr}_{0.8} \mathrm{Ce}_{0.2} \mathrm{Mn}_{1-y} \mathrm{Co}_{y} \mathrm{O}_{3-\delta}$ интересно с точки зрения эволюции кристаллической структуры и конкуренции между антиферромагнитными и ферромагнитными взаимодействиями. В [17] методами рентгеновской абсорбционной спектроскопии и магнитных измерений проанализированы окислительные состояния $\mathrm{Ce}, \mathrm{Mn}$ и Со в ряде $\mathrm{TP} \mathrm{Sr}_{1-x} \mathrm{Ce}_{x} \mathrm{Mn}_{1-y} \mathrm{Co}_{y} \mathrm{O}_{3-\delta}$.

\section{2. Эксперимент}

Синтез образцов $\mathrm{Sr}_{0.8} \mathrm{Ce}_{0.2} \mathrm{Mn}_{1-y} \mathrm{Co}_{y} \mathrm{O}_{3-\delta}$ осуществляли методом твердофазных реакций из простых оксидов $\mathrm{CeO}_{2}, \mathrm{MnO}_{2}, \mathrm{Co}_{3} \mathrm{O}_{4}$ и $\mathrm{SrCO}_{3}$, содержащих не менее 99.95\% основного вещества. Стехиометрические смеси указанных оксидов и карбона стронция тщательно перетирались, прессовались под давлением $3000 \mathrm{~kg} / \mathrm{cm}^{2}$ и спекались в режиме ступенчатого повышения температуры с шагом $100^{\circ} \mathrm{C}$ и промежуточными перетираниями через каждые 10 часов. Продукты реакций после прокаливания охлаждались вместе с печью до комнатной температуры. Начальная температура отжига - $950^{\circ} \mathrm{C}$, заключительная - $1350^{\circ} \mathrm{C}$. Наличие примесей в продуктах контролировали рентгенографически на дифрактометре Shimadzu XRD-7000 S с использованием базы данных PDF 2 (ICDD, USA, Release 2009). Обработку дифрактограмм и уточнение кристаллохимических параметров проводили по методу полнопрофильного анализа Ритвелда в программе FULLPROF 2016.

Содержание кислорода в образцах анализировали по убыли массы при прокаливании в токе $\mathrm{H}_{2}$ при $950^{\circ} \mathrm{C}$ в течение четырех часов. Индекс при кислороде в исходных образцах рассчитывали с учетом того, что Со в процессе реакции восстанавливается до металлического состояния, а оставшийся кислород связан с двухвалентным марганцем, трехвалентным церием и $\mathrm{SrO}$.

Магнитные измерения проводили в центре коллективного использования ИФМ УрО РАН на СКВИД магнитометре MPMS--XL-5 фирмы QUANTUM DESIGN в интервале температур $2-300 \mathrm{~K}$ в магнитных полях 0.5 и $5.0 \mathrm{kOe}(D C$-восприимчивость). Измерения производились в режимах охлаждения образцов в измеряемом $(F C$-данные) и нулевом ( $Z F C$-данные) магнитных полях. Используя метод измерения динамической магнитной восприимчивости ( $A C$-восприимчивости), определяли действительную $\chi^{\prime}$ и мнимую $\chi^{\prime \prime}$ составляющие восприимчивости при амплитудном значении переменного магнитного поля до 4 Ое и при частоте $80 \mathrm{~Hz}$. Кривые намагничивания и петли магнитного гистерезиса были получены при $2 \mathrm{~K}$ на образцах, охлажденных в нулевом магнитном поле, по следующей схеме: сначала измеряли кривую намагничивания, увеличивая магнитное поле от 0 до $+50 \mathrm{kOe}$, а затем выполнялись измерения петли гистерезиса при измении поля от +50 до $-50 \mathrm{kOe}$ и от -50 до $+50 \mathrm{kOe}$.

\section{3. Результаты и обсуждение}

\section{1. Структурные характеристики TP $\mathrm{Sr}_{0.8} \mathrm{Ce}_{0.2} \mathrm{Mn}_{1-y} \mathrm{Co}_{y} \mathrm{O}_{3-\delta}(y=0.2$, $0.3,0.4,0.5$ и 0.6 )}

По данным рентгенографических исследований все образцы при значениях $y$ в диапазоне $0.2-0.6$ являются однофазными. Состав с $y=0.7$ содержит наряду с 
Таблица 1. Структурные параметры и содержание кислорода в твердых растворах $\mathrm{Sr}_{0.8} \mathrm{Ce}_{0.2} \mathrm{Mn}_{1-y} \mathrm{Co}_{y} \mathrm{O}_{3-\delta}$

\begin{tabular}{l|c|c|c|c|c}
\hline$y$ & $\begin{array}{c}\text { Пространственная } \\
\text { группа }\end{array}$ & $a, \AA$ & $c, \AA$ & $V, \AA^{3}$ & $\delta$ \\
\hline $0[8]$ & $i 4 / m c m$ & $5.4013(1)$ & $7.7481(1)$ & 225.95 & 0 \\
0.2 & $I 4 / m c m$ & $5.3962(1)$ & $7.6674(1)$ & $223.22(1)$ & 0.04 \\
0.3 & $I 4 / m c m$ & $5.3953(1)$ & $7.6487(1)$ & $222.64(1)$ & 0.06 \\
0.4 & $P m 3 m$ & $3.8222(1)$ & & 55.83 & 0.12 \\
0.5 & $P m 3 m$ & $3.8292(1)$ & & 56.15 & 0.25 \\
0.6 & $P m 3 m$ & $3.8406(1)$ & & 56.65 & 0.31
\end{tabular}

основной фазой оксид церия $\mathrm{CeO}_{2}$. Полученные нами параметры кристаллической структуры для $\mathrm{Sr}_{0.8} \mathrm{Ce}_{0.2} \mathrm{MnO}_{3}$ практически соответствуют опубликованным в работе [8]. В табл. 1 приведены параметры $a$ и $c$ ТР $\mathrm{Sr}_{0.8} \mathrm{Ce}_{0.2} \mathrm{Mn}_{1-y} \mathrm{Co}_{y} \mathrm{O}_{3}(y=0-0.6)$ и содержание кислорода в образцах. Установлено, что ТР с $y=0.2$ и 0.3 , как и $\mathrm{Sr}_{0.8} \mathrm{Ce}_{0.2} \mathrm{MnO}_{3}$, являются тетрагональными (пространственная группа $I 4 / \mathrm{mcm}$. Образцы с $y \geq 0.4$ имеют кубическую ячейку (пространственная группа $P \mathrm{~m} 3 \mathrm{~m}$ ). Таким образом, ТР при $y \geq 0.4$ характеризуются фазовым переходом из тетрагональной сингонии в кубическую, которая устойчива для ТР с $y=0.5$ и 0.6. Уменьшение содержания кислорода в ТP с по мере замещения $\mathrm{Mn}$ на Со свидетельствует об образовании дефицитных по кислороду образцов, что вполне закономерно и согласуется с составом кобальтита $\mathrm{Sr}_{0.9} \mathrm{Ce}_{0.1} \mathrm{CoO}_{3.69}$ [12].

На рис. 1 приведены зависимости от $y$ параметров $a$ и $c$ и объема $V$ элементарной ячейки ТР $\mathrm{Sr}_{0.8} \mathrm{Ce}_{0.2} \mathrm{Mn}_{1-y} \mathrm{Co}_{y} \mathrm{O}_{3-\delta}$. Значения $a, c$ и $V$ для ТР с $y=0,0.2$ и 0.3 на рис. 1 даны для сравненения для простой ячейки перовскита. Усредненный параметр $V_{0}$ для тетрагональных ТР был вычислен по формуле $V_{0}=a^{2} c / 4$. Видно, что при увеличении степени замещения $\mathrm{Mn}$ на Со наблюдается существенное изменение параметров кристаллической решетки. Введение Со в

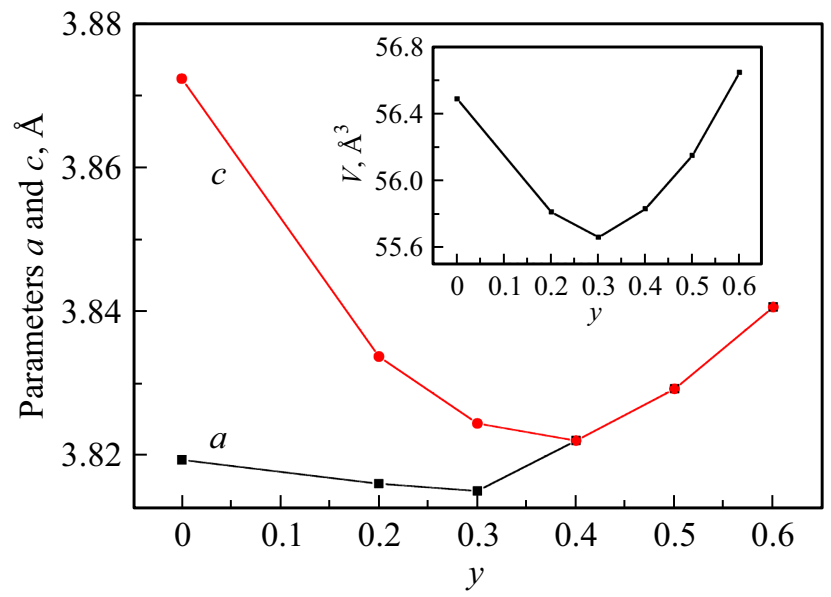

Рис. 1. Зависимость параметров $a, c$ и объема $V$ (на вставке) для $\mathrm{Sr}_{0.8} \mathrm{Ce}_{0.2} \mathrm{Mn}_{1-y} \mathrm{Co}_{y} \mathrm{O}_{3-\delta}$ от содержания кобальта. кислородный октаэдр приводит, прежде всего, к резкому уменьшению параметра $c$, причиной которого является снижение количества катионов $\mathrm{Mn}^{3+}$. Параметр $a$ в интервале от 0 до 0.3 уменьшается в меньшей степени.

Объем элементарной ячейки ТР с тетрагональной структурой в данном интервале значений $y$ также уменьшается по мере увеличения содержания кобальта. При $y>0.3$ и в кубических ТР параметр $a$ и объем $V$ возрастают. Твердый раствор с максимальным содержанием $\mathrm{Co}\left(\mathrm{Sr}_{0.8} \mathrm{Ce}_{0.2} \mathrm{Mn}_{0.4} \mathrm{Co}_{0.6} \mathrm{O}_{2.69}\right)$ имеет параметр $a(3.841 \AA)$, близкий к параметру $\mathrm{Sr}_{0.9} \mathrm{Ce}_{0.1} \mathrm{CoO}_{2.74}$ $(a=3.843 \AA[12])$.

\section{2. Магнитные свойства твердых растворов $\mathrm{Sr}_{0.8} \mathrm{Ce}_{0.2} \mathrm{Mn}_{1-y} \mathrm{Co}_{y} \mathrm{O}_{3-\delta}$}

Результаты измерений магнитных характеристик ТР $\mathrm{Sr}_{0.8} \mathrm{Ce}_{0.2} \mathrm{Mn}_{1-y} \mathrm{Co}_{y} \mathrm{O}_{3-\delta}$ в интервале $2-300 \mathrm{~K}$ приведены на рис. $2-5$. Температурные зависимости магнитной восприимчивости $\chi$ всех исследованных при $H=5 \mathrm{kOe}$ образцов характеризуются наличием двух аномалий (рис. 2 и 3) и уменьшением $\chi$ с увеличением содержания Со. Высокотемпературные аномалии $T_{\max 1}$ при всех значениях $y$ отвечают переходу от парамагнитного к некоторому новому магнитному состоянию. Тот факт, что магнитная восприимчивость, измеренная в режиме $\mathrm{FC}$, не имеет тенденции к резкому увеличению при температурах ниже $T_{\max 1}$, позволяет сделать вывод об отсутствии ферромагнитного упорядочения во всех изученных ТР. Ниже этих максимумов появляется расхождение между кривыми $\mathrm{FC}$ и ZFC, резко увеличивающееся при $T_{\max 2}$ (при $20-40 \mathrm{~K}$ ). Расхождение между кривыми $\mathrm{FC}$ и ZFC ниже точки магнитного превращения $T_{\max } 1$ характерно для всех образцов, содержащих Со. Измерения в магнитном поле 0.5 и $5 \mathrm{kOe}$ совпадают, за исключением ТР с $y=0.6$. Как следует из рис. 4 , на температурной зависимости $\chi \mathrm{Sr}_{0.8} \mathrm{Ce}_{0.2} \mathrm{Mn}_{0.4} \mathrm{Co}_{0.6} \mathrm{O}_{2.69}$, измеренной в поле $H=0.5 \mathrm{kOe}$, наблюдается еще одна аномалия, сопровождающаяся резким расхождением кривых ZFC и FC. Эта аномалия, сопровождаемая небольшими максимумами на кривых ZFC при $136 \mathrm{~K}$ в поле 500 Ое, наиболее выражена и смещена до $142 \mathrm{~K}$ при измерении в магнитном поле 100 Ое (вставка на рис. 4).

При минимальном замещении $\mathrm{Mn}$ на Со $(y=0.2)$ ТР характеризуется, в сравнении с $\mathrm{Sr}_{0.8} \mathrm{Ce}_{0.2} \mathrm{MnO}_{3}$ [8], менее выраженным широким максимумом на зависимости $\chi(T)$ в интервале $100-160 \mathrm{~K}$ (рис. 2). На температурной зависимости магнитной восприимчивости ниже $138 \mathrm{~K}$ наблюдается расхождение кривых ZFC и $\mathrm{FC}$, а зависимость $\chi(T)$ не имеет излома, характерного для АФМперехода, связанного со снижением магнитной восприимчивости. В то же время зависимость $\chi^{-1}(T)$ (рис. 5) следует закону Кюри-Вейсса значительно выше $T_{\max } 1$ $(138 \mathrm{~K})$ и имеет положительное значение константы $\Theta$ в интервале $250-400 \mathrm{~K}$, как и в $\mathrm{Sr}_{0.8} \mathrm{Ce}_{0.2} \mathrm{MnO}_{3}$ [8]. 


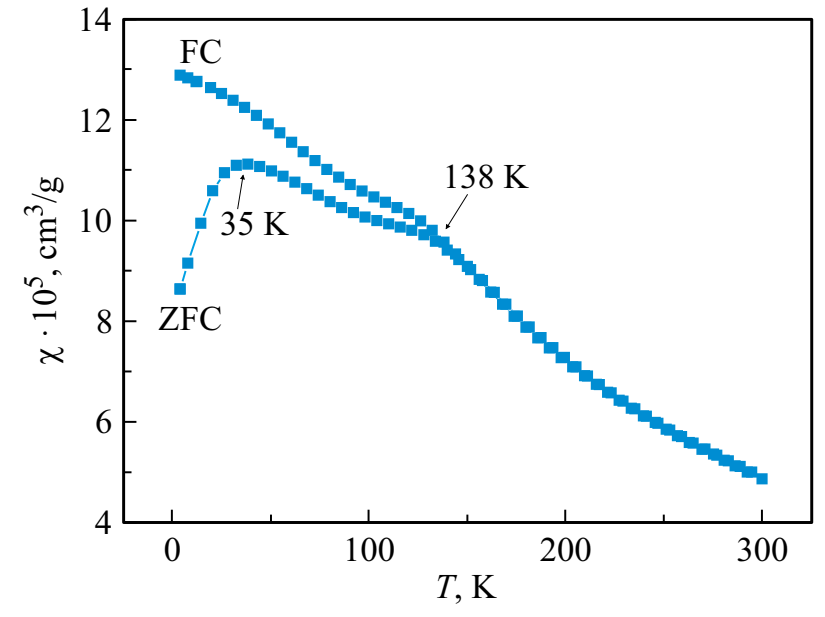

Рис. 2. Температурная зависимость магнитной восприимчивости $\chi$ для $\mathrm{Sr}_{0.8} \mathrm{Ce}_{0.2} \mathrm{Mn}_{0.8} \mathrm{Co}_{0.2} \mathrm{O}_{2.96}$ в магнитном поле $5 \mathrm{kOe}$.

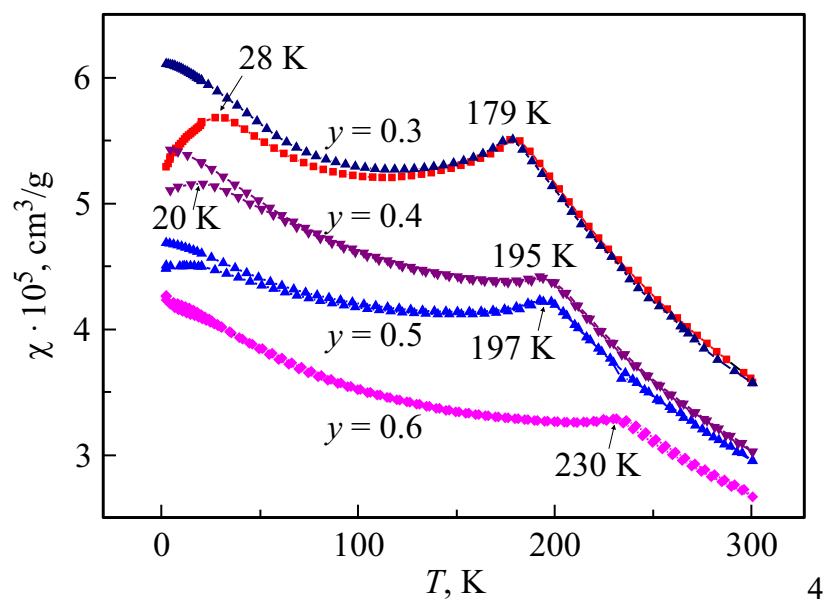

Рис. 3. Температурная зависимость магнитной восприимчивости $\chi$ для $\mathrm{Sr}_{0.8} \mathrm{Ce}_{0.2} \mathrm{Mn}_{1-y} \mathrm{Co}_{y} \mathrm{O}_{3-\delta}(y=0.3-0.6)$ в магнитном поле $5 \mathrm{kOe}$.

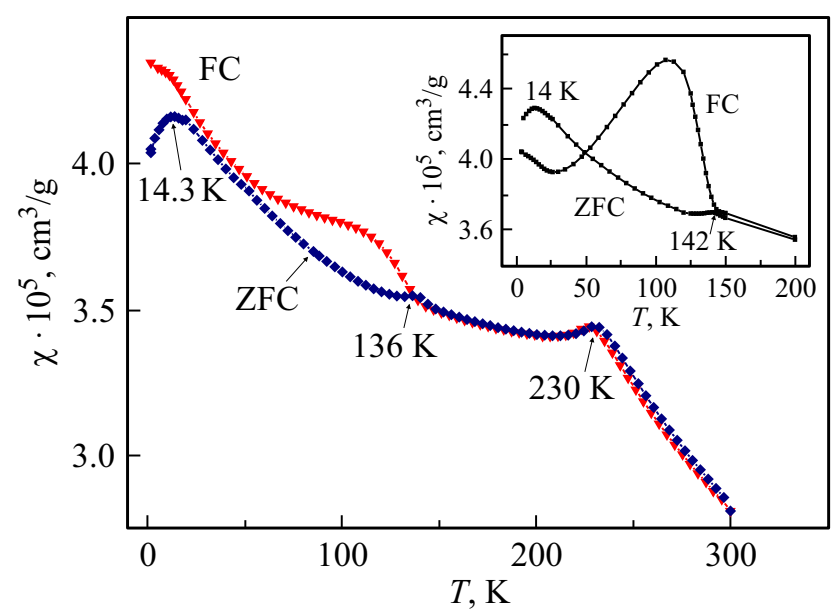

Рис. 4. Температурная зависимость магнитной восприимчивости $\chi$ для $\mathrm{Sr}_{0.8} \mathrm{Ce}_{0.2} \mathrm{Mn}_{0.4} \mathrm{Co}_{0.6} \mathrm{O}_{2.70}$ в магнитных полях 0.5 и 0.1 (на вставке) kOe.
Таблица 2. Магнитные свойства твердых растворов $\mathrm{Sr}_{0.8} \mathrm{Ce}_{0.2} \mathrm{Mn}_{1-y} \mathrm{Co}_{y} \mathrm{O}_{3-\delta}$

\begin{tabular}{l|c|c|c|c}
\hline$y$ & $\Theta, \mathrm{K}$ & $C, \mathrm{emu} \mathrm{K} / \mathrm{mol}$ & $T_{g} \mathrm{~K}$ & $T_{N}, \mathrm{~K}$ \\
\hline 0.2 & 11 & 2.83 & 35 & 138 \\
0.3 & -34.7 & 2.42 & 28 & 179 \\
0.4 & -39.4 & 2.0 & 20 & 195 \\
0.5 & -35.6 & 1.96 & 16 & 197 \\
0.6 & -80 & 1.98 & 12 & 230
\end{tabular}

В ТР с более высоким содержанием кобальта максимум при температуре $T_{\max 1}$ становится более отчетливым и сдвигается в сторону высоких температур до 179 , 195,197 и $230 \mathrm{~K}$ для $y=0.3,0.4,0.5$ и 0.6 соответственно (рис. 3). При значениях $y=0.3,0.4$ и 0.5 по мере дальнейшего охлаждения в интервале $75-100 \mathrm{~K}$ имеет место небольшое повышение магнитной восприимчивости, сопровождающееся расхождением кривых FC и ZFC. При $28 \mathrm{~K}(y=0.3), 20 \mathrm{~K}(y=0.4)$ и $15 \mathrm{~K}(y=0.5)$ на зависимостях ZFC фиксируются максимумы. Как следует из рис. 5 , при высоких температурах магнитная восприимчивость ТР с $x$, равным $0.3,0.4,0.5$ и 0.6 , следует закону Кюри-Вейсса

$$
\chi=C /(T-\Theta),
$$

где $C$ - константа Кюри $\left(\mathrm{cm}^{3}\right)$ и $\Theta-$ константа КюриВейса $(\mathrm{K})$. Отрицательные значения $\Theta$ свидетельствуют о преобладающих антиферромагнитных взаимодействиях. Твердый раствор с $x=0.2$ характеризуется, подобно $\mathrm{Sr}_{0.8} \mathrm{Ce}_{0.2} \mathrm{MnO}_{3}$, положительным значением константы $\Theta$ (табл. 2).

Кривые намагничивания при $2 \mathrm{~K}$ (рис. 6) характеризуются наличием узких петель гистерезиса без признаков насыщения в магнитном поле $50 \mathrm{kOe}$ с остаточной намагниченностью до $0.02 \mathrm{emu} / \mathrm{g}$ и коэрцетивной силой $B$

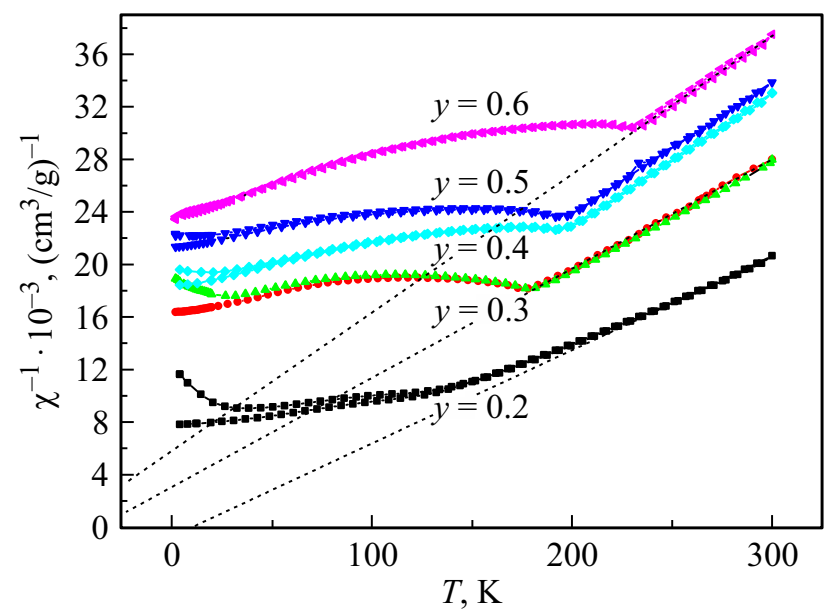

Рис. 5. Температурная зависимость обратной магнитной восприимчивости $\chi^{-1}(T)$ для $\mathrm{Sr}_{0.8} \mathrm{Ce}_{0.2} \mathrm{Mn}_{1-y} \mathrm{Co}_{y} \mathrm{O}_{3-\delta}$ $(y=0.2-0.6)$ при $5 \mathrm{kOe}$. 
до 350 Ое. С учетом этих данных низкотемпературные аномалии в $\mathrm{TP} \mathrm{Sr}_{0.8} \mathrm{Ce}_{0.2} \mathrm{Mn}_{1-y} \mathrm{Co}_{y} \mathrm{O}_{3-\delta}$ при 20-34, так же как в случае $\mathrm{Sr}_{0.8} \mathrm{Ce}_{0.2} \mathrm{MnO}_{3}$ [9], должны быть отнесены к переходам в спин-стекольное состояние.

Согласно измерениям АС-восприимчивости ряда образцов (рис. 7), на кривых $\chi^{\prime}(T)$ присутствуют аномалии при температурах, близких к $T_{\max 1}$ и $T_{\max 2}$, зафиксированных при измерении DC восприимчивости.

В табл. 2 и 3 приведены магнитные характеристики всех изученных образцов. Сведения об образце с значением $y=0.2$ получены исходя из зависимости $\chi^{-1}(T)$ в интервале $250-400 \mathrm{~K}$ [16]. По данным спектроскопии RAS и XANES, приведенным в работах $[15,17]$, церий в данных ТР находится в виде катионов $\mathrm{Ce}^{4+}$, следовательно, суммарный магнитный момент приходится на катионы кобальта и марганца. Расчет валентных и спиновых состояний $\mathrm{Mn}$ и $\mathrm{Co}$ в $\mathrm{Sr}_{0.8} \mathrm{Ce}_{0.2} \mathrm{Mn}_{1-y} \mathrm{Co}_{y} \mathrm{O}_{3-\delta}$ $(y=0.2-0.6)$ проводили с учетом сведений о кислородной нестехиометрии образцов (табл. 1) и экспериментально определенных эффективных магнитных мо-
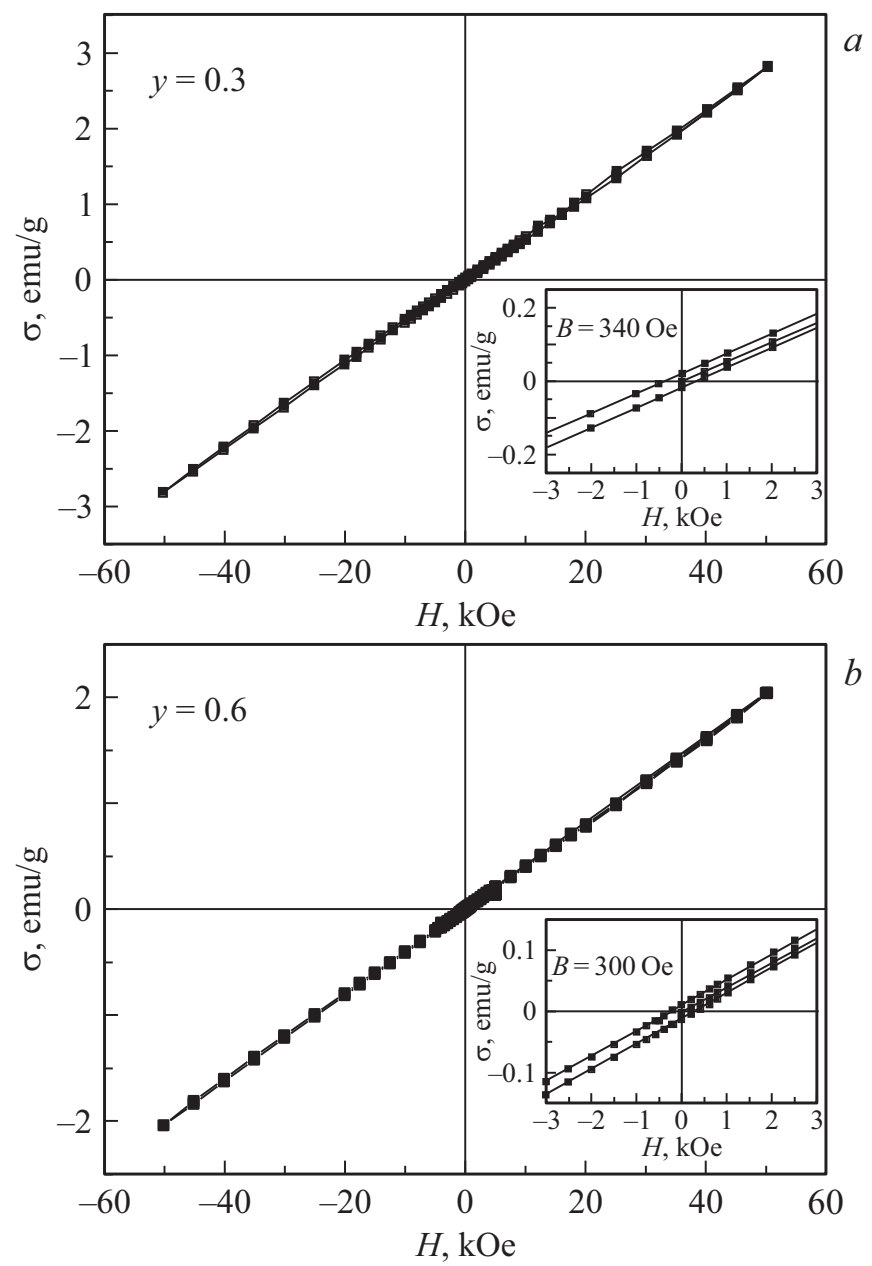

Рис. 6. Зависимость намагниченности $\sigma$ от приложенного магнитного поля для $a) \mathrm{Sr}_{0.8} \mathrm{Ce}_{0.2} \mathrm{Mn}_{0.7} \mathrm{Co}_{0.3} \mathrm{O}_{2.94}(y=0.3)$ и $b) \mathrm{Sr}_{0.8} \mathrm{Ce}_{0.2} \mathrm{Mn}_{0.4} \mathrm{Co}_{0.6} \mathrm{O}_{2.69}(y=0.6)$ при $2 \mathrm{~K}$.

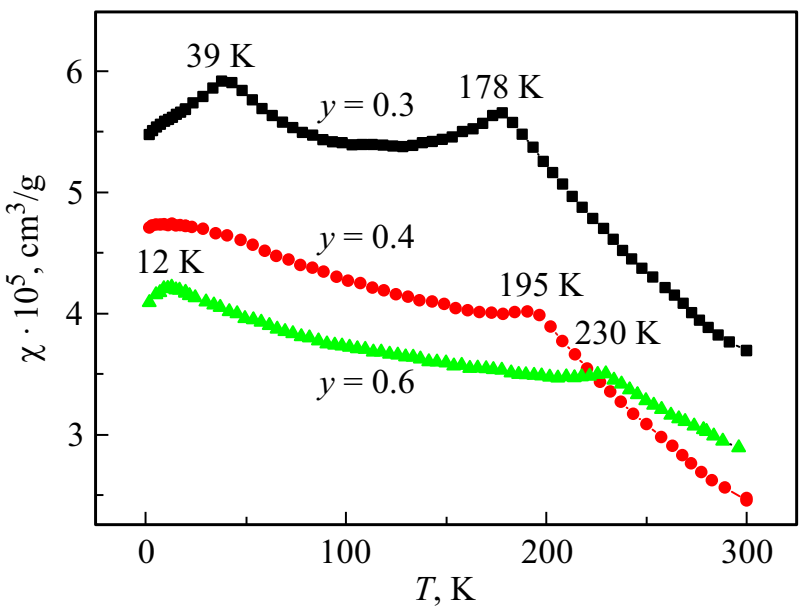

Рис. 7. Температурная зависимость АС магнитной восприимчивости $\chi^{\prime}$ для $\mathrm{Sr}_{0.8} \mathrm{Ce}_{0.2} \mathrm{Mn}_{1-y} \mathrm{Co}_{y} \mathrm{O}_{3-\delta}$ при $y$, равном 0.3, 0.4 и 0.6 .

ментов. Для расчетов были использованы следующие конфигурации катионов в октаэдрическом окружении: $\mathrm{Mn}^{4+}\left(t_{2 g}^{3} e_{g}^{0}, S=3 / 2\right), \mathrm{Mn}^{3+}\left(t_{2 g}^{3} e_{g}^{1}, S=2\right), \mathrm{Co}^{2+}\left(t_{2 g}^{5} e_{g}^{2}\right.$, $S=3 / 2), \mathrm{Co}^{3+}\left(t_{2 g}^{4} e_{g}^{2}, S=2\right.$ - высокоспиновое состояние). Учитывался также спин орбитальный вклад в эффективный магнитный момент $\mathrm{Co}^{2+}$.

Из данных табл. 2 следует, что экспериментально найденный эффективный магнитный момент $\mu_{\text {exp }}\left(4.76 \mu_{B}\right)$ для $\mathrm{TP} \mathrm{Sr}_{0.8} \mathrm{Ce}_{0.2} \mathrm{Mn}_{0.8} \mathrm{Co}_{0.2} \mathrm{O}_{3}$ значительно выше вычисленного $\mu_{\text {calc }}$ в предположении, что марганец находится в виде катионов $\mathrm{Mn}^{4+}$, а кобальт - в виде $\mathrm{Co}^{2+}$. Между тем, ранее в работе [15] методом спектроскопии XANES было показано, что $\mathrm{Mn} \mathrm{в} \mathrm{Sr}_{0.8} \mathrm{Ce}_{0.2} \mathrm{Mn}_{0.8} \mathrm{Co}_{0.2} \mathrm{O}_{3}$ имеет более высокую валентность, чем в $\mathrm{Sr}_{0.8} \mathrm{Ce}_{0.2} \mathrm{MnO}_{3}$. Это означает, что большая часть Мn в данном ТР. находится в виде катионов $\mathrm{Mn}^{4+}$, а Со в виде катионов $\mathrm{Co}^{2+}$. Этот вывод согласуется с результатами исследований других сложных оксидов $\mathrm{Mn}$ и Со, в частности, со структурами перовскита [6] и шпинели [18], в которых также зафиксирована устойчивая валентная конфигурация $\mathrm{Mn}^{4+}-\mathrm{Co}^{2+}$, а не $\mathrm{Mn}^{3+}-\mathrm{Co}^{3+}$. Повышенное значе-

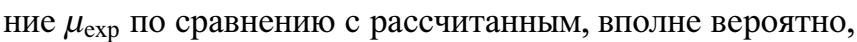
следует связать, как и в случае с $\mathrm{Sr}_{0.8} \mathrm{Ce}_{0.2} \mathrm{MnO}_{3}$, с образованием ферромагнитных кластеров в парамагнитной области.

Как следует из табл. 2, при дальнейшем увеличении содержания Со в ТР количество катионов $\mathrm{Mn}^{3+}$ уменьшается (до $16 \%$ при $y=0.3$ ) и при $y$, равном 0.4 и 0.5 , они полностью исчезают. В результате уменьшения содержания кислорода в этих образцах увеличивается содержание двухвалентного кобальта.

Представляет интерес сравнить рассмотренную эволюцию валентного состояния Со и $\mathrm{Mn} \mathrm{c} \mathrm{известными}$ данными о системе $\mathrm{SrMn}_{1-x} \mathrm{Co}_{x} \mathrm{O}_{3}$. В данной системе, в отличие от $\mathrm{Sr}_{0.8} \mathrm{Ce}_{0.2} \mathrm{Mn}_{1-x} \mathrm{Co}_{x} \mathrm{O}_{3}$, замещение $\mathrm{Mn}$ на Co приводит к образованию ряда гомологических соеди- 
Таблица 3. Экспериментальные и вычисленые магнитные моменты $\mathrm{Mn}$ и Со в твердых растворах $\mathrm{Sr}_{0.8} \mathrm{Ce}_{0.2} \mathrm{Mn}_{1-y} \mathrm{Co}_{y} \mathrm{O}_{3-y}$

\begin{tabular}{|c|c|c|c|}
\hline$y$ & Состав & $\mu_{\mathrm{calc}}, \mu_{B}$ & $\mu_{\exp }, \mu_{B}$ \\
\hline 0 & $\mathrm{Sr}_{0.8} \mathrm{Ce}\left(\mathrm{IV}_{0.2}\right) \mathrm{Mn}\left(\mathrm{IV}_{0.6}\right) \mathrm{Mn}(\mathrm{III})_{0.4} \mathrm{O}_{3}$ & 4.31 & $5.96[8]$ \\
\hline 0.2 & $\mathrm{Sr}_{0.8} \mathrm{Ce}_{0.2} \mathrm{Mn}(\mathrm{IV})_{0.72} \mathrm{Mn}(\mathrm{III})_{0.08} \mathrm{Co}(\mathrm{II})_{0.02} \mathrm{O}_{2.96}$ & 4.27 & 4.76 \\
\hline 0.3 & $\mathrm{Sr}_{0.8} \mathrm{Ce}_{0.2} \mathrm{Mn}(\mathrm{IV})_{0.60} \mathrm{Mn}(\mathrm{III})_{0.10} \mathrm{Co}(\mathrm{II})_{0.20} \mathrm{Co}\left(\mathrm{III}_{\mathrm{HS}}\right)_{0.10} \mathrm{O}_{2.94}$ & 4.38 & 4.40 \\
\hline 0.4 & $\mathrm{Sr}_{0.8} \mathrm{Ce}_{0.2} \mathrm{Mn}(\mathrm{IV})_{0.60} \mathrm{Co}(\mathrm{II})_{0.24} \mathrm{Co}\left(\mathrm{III}_{\mathrm{HS}}\right)_{0.04} \mathrm{Co}\left(\mathrm{III}_{\mathrm{LS}}\right)_{0.12} \mathrm{O}_{2.88}$ & 4.06 & 4.04 \\
\hline 0.5 & $\mathrm{Sr}_{0.8} \mathrm{Ce}_{0.2} \mathrm{Mn}(\mathrm{IV})_{0.5} \mathrm{Co}(\mathrm{II})_{0.25} \mathrm{Co}\left(\mathrm{III}_{\mathrm{HS}}\right)_{0.07} \mathrm{Co}\left(\mathrm{III}_{\mathrm{LS}}\right)_{0.18} \mathrm{O}_{2.75}$ & 3.99 & 3.96 \\
\hline 0.6 & $\mathrm{Sr}_{0.8} \mathrm{Ce}_{0.2} \mathrm{Mn}(\mathrm{IV})_{0.40} \mathrm{Co}(\mathrm{II})_{0.40} \mathrm{Co}\left(\mathrm{III}_{\mathrm{HS}}\right)_{0.07} \mathrm{Co}\left(\mathrm{III}_{\mathrm{LS}}\right)_{0.20} \mathrm{O}_{2.69}$ & 4.10 & 4.01 \\
\hline
\end{tabular}

нений с квазиодномерной структурой: $\mathrm{Sr}_{14} \mathrm{Mn}_{8} \mathrm{Co}_{3} \mathrm{O}_{33}$, $\mathrm{Sr}_{9} \mathrm{Mn}_{5} \mathrm{Co}_{2} \mathrm{O}_{21}$ и $\mathrm{Sr}_{4} \mathrm{Mn}_{2} \mathrm{CoO}_{9}$ [19]. В этих соединениях $d$-элементы упорядочены по разным структурным позициям в виде катионов $\mathrm{Mn}^{4+}$ и $\mathrm{Co}^{2+}$. Присутствие катионов $\mathrm{Mn}^{4+}$ рассматривается в качестве наиболее вероятной ситуации в $\mathrm{TP} \mathrm{SrCo}_{1-x} \mathrm{Mn}_{x} \mathrm{O}_{3-\delta}$ $(0 \leq x \leq 0.30)$ [20]. Из табл. 3 следует, что в пределах $\mathrm{TP} \mathrm{Sr}_{0.8} \mathrm{Ce}_{0.2} \mathrm{Mn}_{1-y} \mathrm{Co}_{y} \mathrm{O}_{3}$ с ростом унаблюдается тенденция к уменьшению числа катионов $\mathrm{Mn}^{3+}$ и преимущественному образованию валентной комбинации $\mathrm{Mn}^{4+}-\mathrm{Co}^{2+}$.

На основании приведенных в табл. 3 данных можно объяснить изменение параметров элементарной ячейки в ряду твердых растворов $\mathrm{Sr}_{0.8} \mathrm{Ce}_{0.2} \mathrm{Mn}_{1-y} \mathrm{Co}_{y} \mathrm{O}_{3-\delta}$ $(y=0-0.6)$. Уменьшение тетрагонального искажения и параметров $a$ и $c$ на первом этапе коррелирует с понижением содержания ян-теллеровских катионов $\mathrm{Mn}^{3+}$ (с ионным радиусом $0.645 \AA[21]$ ), которые замещаются катионами $\mathrm{Co}^{3+}$, имеющими меньшие размеры $(0.61 \AA)$. В дальнейшем определяющий вклад в размеры элементарной ячейки и переход к кубической структуре вносят катионы $\mathrm{Co}^{2+}$ (ионный радиус $0.745 \AA$ ).

Для оценки природы магнитных взаимодействий в изученных твердых растворах были приняты во внимание данные работ $[8,9]$ о том, что $\mathrm{Sr}_{0.8} \mathrm{Ce}_{0.2} \mathrm{MnO}_{3}$ характеризуется как разбавленный антиферромагнетик $\left(T_{N}=210 \mathrm{~K}\right)$ со спинстекольным состоянием в области ниже $20 \mathrm{~K}$ и с ферромагнитными кластерами или микроскопической негомогенной магнитной фазой в парамагнитной области. Анализ валентного и спинового состояния $\mathrm{Mn}$ и $\mathrm{Co}$ в $\mathrm{Sr}_{0.8} \mathrm{Ce}_{0.2} \mathrm{Mn}_{1-y} \mathrm{Co}_{y} \mathrm{O}_{3-\delta}(y=0-0.6)$ на основе магнитных измерений позволяет утверждать, что возможными антиферромагнитными (AFM) взаимодействиями в рассмотренных композициях являются $\mathrm{Mn}^{3+}-\mathrm{O}-\mathrm{Mn}^{3+}, \mathrm{Mn}^{4+}-\mathrm{O}-\mathrm{Mn}^{4+}$ и $\mathrm{Co}^{3+}-\mathrm{O}-\mathrm{Mn}^{4+}$, а ферромагнитными взаимодействиями (FM) $\mathrm{Co}^{2+}-\mathrm{O}-\mathrm{Mn}^{4+}$ и $\mathrm{Mn}^{3+}-\mathrm{O}-\mathrm{Mn}^{4+}$. В $\mathrm{Sr}_{0.8} \mathrm{Ce}_{0.2} \mathrm{MnO}_{3}$ магнетизм определяется конкуренцией между взаимодействиями $\mathrm{Mn}^{3+}-\mathrm{O}-\mathrm{Mn}^{3+}$ и $\mathrm{Mn}^{4+}-\mathrm{O}-\mathrm{Mn}^{4+}$ и взаимодействиями $\mathrm{Mn}^{3+}-\mathrm{O}-\mathrm{Mn}^{4+}$. Смещение широкого максимума в $\mathrm{Sr}_{0.8} \mathrm{Ce}_{0.2} \mathrm{Mn}_{0.8} \mathrm{Co}_{0.2} \mathrm{O}_{2.95}$ до $138 \mathrm{~K}$ свидетельствует об ослаблении антиферромагнитных корреляций ближнего порядка при сохранении сложной зависимости $\chi^{-1}(T)$ выше этой температуры. Положительное значение константы $\Theta$ для этого образца и существенное расхождение между кривыми ZFC и FC ниже $35 \mathrm{~K}$ указывают на сохранение конкурирующих ферромагнитных и антиферромагнитных взаимодействий, следствием которых является спин-стекольное состояние при низких температурах. Повышение $T_{\max 1}$ при увеличении содержания Со (до $239 \mathrm{~K}$ при $y=0.6$ ) и рост отрицательных значений константы $\Theta$ происходят, по видимому, за счет усиления АФМ взаимодействий $\mathrm{Mn}^{4+}-\mathrm{O}-\mathrm{Mn}^{4+}$ на фоне ослабления ФМ взаимодействий. Появление скачка $\chi$ на $\mathrm{FC}$-кривой и слабый максимум на ZFC-кривой зависимости $\chi(T)$ при $140 \mathrm{~K}$ в образце $\mathrm{Sr}_{0.8} \mathrm{Ce}_{0.2} \mathrm{Mn}_{0.4} \mathrm{Co}_{0.6} \mathrm{O}_{2}$ при измерении в магнитных полях 500 и 100 Ое свидетельствуют о магнитной неоднородности данного образца и обусловлены высоким содержанием катионов $\mathrm{Co}^{2+}$ и образовании ФМ кластеров $\mathrm{Co}^{2+}-\mathrm{Mn}^{4+}$. Данный вывод может быть подтвержден результатами исследований ТP $\mathrm{SrCo}_{1-x} \mathrm{Mn}_{x} \mathrm{O}_{2.74}$, в котором ферромагнетизм с $T_{C}=150 \mathrm{~K}$, обнаруженный при минимальном содержании $\mathrm{Mn}(x=0.05-0.10)$, при большем содержании этого элемента разрушается [20].

\section{4. Заключение}

Результаты исследования кристаллической структуры и измерений магнитных характеристик электрон допированных $\mathrm{TP} \mathrm{Sr}_{0.8} \mathrm{Ce}_{0.2} \mathrm{Mn}_{1-y} \mathrm{Co}_{y} \mathrm{O}_{3-\delta} \quad(y=0.2,0.3$, $0.4,0.5,0.6)$ позволяют сделать следующие выводы. В пределах ТР при $y \geq 0.4$ имеет место концентрационный фазовый переход от тетрагональной сингонии (пространственная группа $I 4 / \mathrm{mcm}$ ) в кубическую (пространственная группа $P m 3 m)$, которая устойчива до значений $y=0.7$. Наблюдаемое структурное превращение связано с замещением ян-теллеровских ионов $\mathrm{Mn}^{3+}$ на катионы кобальта $\mathrm{Co}^{2+}$ и $\mathrm{Co}^{3+}$ и уменьшением содержания кислорода. Эволюция магнитных свойств ТР проанализирована на основе известных данных о манганите $\mathrm{Sr}_{0.8} \mathrm{Ce}_{0.2} \mathrm{MnO}_{3}$, который охарактеризован как разбавленный антиферромагнетик с $T_{N} 250 \mathrm{~K}$ со спинстекольным поведением при низких температурах, содержащий ферромагнитные кластеры в парамагнитной области. При минимальном замещении марганца на кобальт $(y=0.2)$ в $\mathrm{Sr}_{0.8} \mathrm{Ce}_{0.2} \mathrm{MnO}_{3}$ наблюдается резкое уменьшение $T_{N}$ до $138 \mathrm{~K}$, сохранение положительного значения константы $\Theta$ в законе Кюри-Вейса и спинстекольное поведение при $T<35 \mathrm{~K}$. Поведение об- 
разцов с большим содержанием кобальта обусловлено дальнейшим уменьшением содержания катионов $\mathrm{Mn}^{3+}$ (при $y=0.3$ ) и их исчезновением (при $y=0.4$ и 0.5 ). В результате, точка магнитного превращения сдвигается в высокотемпературную область, по-видимому, за счет усиления $\mathrm{Mn}^{4+}-\mathrm{O}-\mathrm{Mn}^{4+}$ АФМ-взаимодействий при сохранении спин-стекольного состояния при низкой температуре. В образце с максимальным содержанием кобальта при измерении в полях 100 и $500 \mathrm{Oe}$ на фоне возможного антиферромагнетизма $\left(T_{N}=230 \mathrm{~K}\right)$ ниже $140 \mathrm{~K}$ обнаружена область ферромагнитных кластеров.

\section{Список литературы}

[1] A. Urushibara, Y. Moritomo, T. Arima, A. Asamitsu, G. Kido, Y. Tokura. Phys. Rev. B 51, 14103 (1995).

[2] M. Imada, A. Fujimori, Y. Tokura. Rev. Mod. Phys. 70, 1039 (1998).

[3] S. Balamurugan, K. Yamaura, A.B. Karki, D.P. Young, M. Arai, E. Takayama Muromachi. Phys. Rev. B 74, 172406 (2006).

[4] J.S. Srikiran, A. Das, P.L. Paulose, S.K. Paranjpe. Appl. Phys. A 74 [Suppl.] S814 (2002).

[5] N. Gayathri, A.K. Raychaudhuri, S.K. Tiwary. Phys. Rev. B 56, 1345 (1997).

[6] G.V. Bazuev, A.V. Korolyov, M.A. Melkozyorova, T.I. Chupakhina. J. Magn. Magn. Mater. 322, 494 (2010).

[7] A. Sundaresan, J.L. Tholence, A. Maignan, C. Martin, M. Hervieu, B. Raveau, E. Suard. Eur. Phys. J. B 14, 431 (2000).

[8] W.J. Lu, B.C. Zhao, R. Ang, W.H. Song, J.J. Du, Y.P. Sun. Phys. Lett. 346, 321 (2005).

[9] P. Mandal, A. Hassen, A. Loidl. Phys.Rev. B 69, 224418 (2004).

[10] H. Gu, H. Chen, L. Gao, Y. Zheng, X. Zhu, L. Guo. Electrochem. Acta. 54, 3532 (2009).

[11] S. Balamurugan, K. Yamaura, M. Arai, E. Takayama Muromachi. Phys. Rev. B 76, 014414 (2007).

[12] A. Maignan, B. Raveau, S. Hebert, V. Pralong, V. Caignaert, D. Pelloquin. J. Phys.: Condens. Matter 18, 4305 (2006).

[13] P.D. Battle, T.C. Gibb, C.W. Jones. J. Solid State Chem. 74, 60 (1988).

[14] H. Wu. K. Zhu, G. Xu, H. Wang. Physica B 407, 770 (2012).

[15] Z. Zhang, C.J. Howard, D. Kennedy, M. Matsuda, M. Miyake. J. Phys.: Condens. Matter. 21, 124218 (2009).

[16] Т.И. Чупахина, Г.В. Базуев. Неорган. материалы 47, 1491 (2011).

[17] S.N. Shamin, V.V. Mesilov, M.S. Udintseva, A.V. Korolev, T.I. Chupakhina, G.V. Bazuev, V.R. Galakhov. Current Appl. Phys. 16, 1597 (2016).

[18] G.V. Bazuev, A.V. Korolyev. J. Magn. Magn. Mater. 320, 2262 (2008).

[19] G.V. Bazuev. Rus. Chem. Rev. 75, 749 (2006).

[20] A. Maignan, S. Hebert, N. Nguyen, V. Pralong, D. Pelloquin, V. Caignaert. J. Magn. Magn. Mater. 303197 (2006).

[21] R.D. Shannon. Acta Cryst. A 32, 75 (1976).

Редактор Е.Ю. Флегонтова 